\title{
Saliva as a Diagnostic Tool in Oral Cancer- An Opinion
}

\author{
Binafsha Manzoor Syed ${ }^{1,2 *}$, Sikander Munir Memon ${ }^{1}$, Fasiha Shah ${ }^{3}$, Shahzad Mughal ${ }^{1}$ and Jawaid Naeem \\ Qureshi ${ }^{4}$ \\ ${ }^{1}$ Medical Research Centre, Liaquat University of Medical \& Health Sciences Jamshoro, Pakistan, \\ ${ }^{2}$ LUMHS Centre, Pakistan Health Research Council, Islamabad, Pakistan \\ ${ }^{3}$ Department of Social Work, Universiti Sains Malaysia \\ ${ }^{4}$ Indus Medical College, Tando Mohammad Khan, Pakistan
}

*Corresponding author: Binafsha Manzoor Syed, Medical Research Centre, Liaquat

University of Medical \& Health Sciences, Jamshoro, Pakistan.

Received Date: December 07, 2020

Published Date: December 18, 2020

Abstract

Testing Saliva is a novel approach and recent research trend in diagnostics of local oral and systemic diseases as liquid biopsy. In patients with oral submucous fibrosis where biopsy further raises the risk of malignant transformation, changes in the composition of saliva including electrolytes, proteins content and presence of RNAs, tumor markers and cells as a panel can potentially be used for early detection of oral cancers.

\section{Introduction}

Saliva is a watery product of salivary glands that maintains healthy oral mucosa by preventing it from drying, lubricating and moistening hard food bolus and helping in its swallowing and also limiting the growth of pathogenic bacteria. Saliva also provides a number of enzymes that initiate food digestion, there by starting carbohydrate and fat metabolism. Saliva is produced mainly by submandibular glands (71\%), parotid (25\%), sublingual (3-4\%) glands and only minute quantity by mucosal and labial glands [1]. The salivary secretions are under control of sympathetic and parasympathetic stimulation producing thick and thin saliva respectively. It has been suggested to be an ultrafiltrate of the blood. It contains a number of enzymes, proteins and biochemicals which come from the blood by ultrafiltration, active transport or diffusion in the salivary glands as well as others added locally such as inflammatory markers, exfoliated cells, immune cells and microorganism as part of normal flora [2]. The water content of the saliva is very high (>99\%) and the quantity of rest of the factors is very low in micro and nanograms. These contents of the saliva indicate local disease and some of them have been suggested as markers of systemic diseases [2]. According to the global epidemiological data oral cancers are among relatively low prevalent cancer (accounting for $3 \%$ of all cancers in men and 2\% in women) [3] as compared to breast and lung cancer, but in countries like Pakistan, India and Bangladesh with high rate of betal nut chewing and smoking oral cancers are climbing high and creating a major health issue. This exposure results in development of inflammatory response leading to chronic lesions and fibrosis with potential risk of malignancy [4]. Oral submucous fibrosis (OSMF) is a precancerous condition with high risk of malignant transformation. Biopsy of OSMF raises the risk of malignancy thus it is not indicated as a result patient are left to develop advanced disease to be diagnosed eventually causes high mortality rate after diagnosis. In a previously reported study including 1774 women of Pakistani origin, out of which $43 \%$ had OSMF only, while $26.6 \%$ has OSMF with malignancy and $30.2 \%$ had cancer without OSMF [5]. By looking at the rate of OSMF with and without cancer it can be said that the more than fifty percentage are at risk of malignant transformation which is quite high. Thus, increasing rate of oral cancers and resulting high mortality warrant alternative methods of diagnosis for oral cancer in patients with established OSMF. In this regard saliva has potential to play a role 
in early diagnosis of cancer. This study prevents a summary of the existing literature and theoretical framework to delineate the potential use of saliva as non-invasive biopsy for oral cancers in patients with OSMF.

\section{Normal Composition of Human Saliva}

Normal saliva is a mixture of almost all the component of plasma including water (>99\%), electrolytes, enzymes, nonenzymatic proteins, immunoglobulins etc. The composition of the saliva changes with variation in the systemic circulation such as it gets thicker by reducing water contents in thirst and dehydration. The $\mathrm{pH}$ of the saliva also varies according to the buffering activity of the food mastication [2]. Similarly, when there is change in blood composition in disease stats there is variation in the composition of saliva. A systematic review of literature conducted recently (i.e.,
2020) including 25 studies, evaluating targeted and non-targeted metabolites in cancer patients. These studies reported at least 140 metabolites out of which most frequently assessed were alanine, valine, leucine. Among breast cancer patient's proline, threonine, and histidine in combination and monoacylglycerol showed high diagnostic potential in breast cancer patients while among patients with oral cancers choline, betaine, pipecolinic acid, and l-carnitine showed better diagnostic potential to determine early stage [6]. There is evidence suggesting altered composition of saliva in oral cancers which was not limited to any one component but there was a significant change in all the constituents ranging from electrolytes to the lipid metabolites and immunoglobulins [7]. Thus, analysis of the saliva could be a potential diagnostic tool in early detection of oral cancers.

\section{Changes in Oral Submucous Fibrosis}

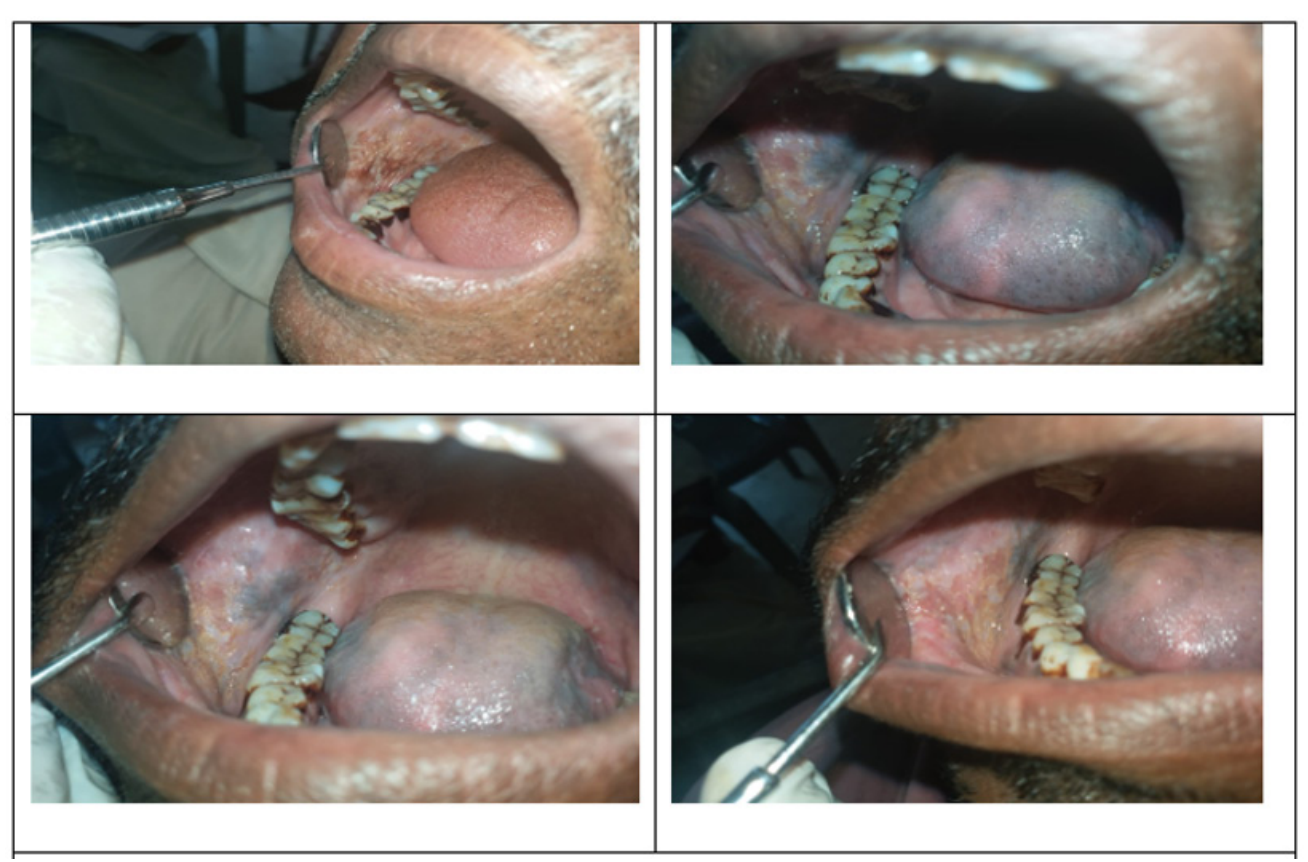

Figure 1: Photographs showing oral submucosal fibrosis in smokeless tobacco chewers- Courtesy: Dr. Sikandar Munir Memon, Medical Research Centre, Liaquat University of Medical \& Health Sciences, Jamshoro, Pakistan.

In cases of smoking and betal nut chewing there are chances of accumulation of toxic chemicals damaging the mucosa [8]. In case of the failure of the antioxidant mechanism of the saliva there is a chance of chronic damage $[9,10]$. The oral sub mucous fibrosis and other pre-malignant lesions have shown to be associated with altered salivary composition which ranges from change in the normal constituents as well as addition of other serum markers such as interleukins and local cellular metabolites. Lactate dehydrogenase (LDH) has been reported to be significantly high in patients with OSMF and oral cancers as compared to controls [11]. Salivary chemerin showed significant difference among controls, patients with pre-malignant oral lesions and cancers. Thus, it has potential to differentiate the malignant lesions from pre-malignant conditions [12]. IL6 and IL8 have been studied and showed a significant potential in differentiating oral pre-malignant lesions from malignancy [13]. Sialic acid showed high concentration among pre-malignant and malignant lesions in the mouth as compared to healthy population [14]. The study on tumor markers including CYFRA-21-1, LDH and total protein were high among premalignant as well as in cancer patients while the comparison of the pre-malignant lesions and cancers was done the concentration was significantly higher among cancer patients [15]. A panel of biochemical components including hydrophilic metabolites, ornithine, o-hydroxybenzoate and ribose 5-Phosphatewere studied in pre-malignant and malignant lesions and reported to show high potential to differentiate between malignant and nonmalignant 
lesions [16]. The study suggested significant rise in the protein content of the saliva in cancer patients, there was also high electrolytes as compared to the normal controls. Immunoglobulins showed high concentration of IgG while lower concentration of IgA [7] (Figure 1).

\section{Changes in Oral cancer}

There are a number of studies which examined saliva in oral cancer patients with and without comparison with controls. A study assessed lipogenese products found a high level of free arachidonic acid and Hydroxy eicosatetraenoic acid (HETE) and high level of linoleic acid in patients with oral cancers [17]. Gene micro-array technique suggested atleast 1679 genes showing varied expression in saliva of patients with oral cancers [18]. Further probing of gene profile and mRNA analysis suggested four mRNA in saliva including OAZ, SAT, IL8, and IL1b showed high potential to diagnose oral cancer early with sensitivity of more than 90\% [19]. Another study focusing on 314 mRNAs found that two mRNAs including miR-125a and miR-200a were significantly lower in patients with oral cancers [20]. Another study has reported deranged level of mRNA 24 and mRNA27b in oral cancers [21]. Yet another study has reported significantly high level of mRNA21, mRNA 184 and mRNA145 in patients with oral cancers [22]. There are other markers such as Ki67 and cyclin D1 which have been studied in multiple cancers, they have also shown significant rise in saliva in patients with oral cancers [23]. The literature suggested high transferrin levels among cancer patient's saliva [24]. Another study evaluated transcriptomes and proteome suggested significant rise in the levels of IL8, IL1B, SAT1 and S100P transcriptomes and IL1B, IL8 and M2BP proteomes in the saliva of patients with oral cancers [25]. Other studies later also confirmed significantly high concentration of IL8 and SAT1 in oral cancer patients [26,27]. The high level of IL6 has also been reported in oral cancer when comparing with precancerous condition [28]. The analysis of the valine, lactic acid and phenylalanine as a combine tool in saliva showed high sensitivity, specificity and positive predictive value in accurately diagnosing oral cancer [29]. Serum tumor markers have also been reported to be raised in the saliva such as CA125 in patients with oral cancer [30]. Salivary e-cadherin has been reported to be very high among cancer patients [31].

Potential role of saliva as a diagnostic tool in oral submucous fibrosis in early detection of oral cancer

The brief review of literature has highlighted efforts being carried out to find a path for early diagnosis of oral cancers in situation of pre-malignant lesions. Looking at the OSMF as taking biopsy rises the risk of malignancy further resulting in high rate of advanced cancer with reduced survival. On the other hand, it is relatively easy to evaluate composition of saliva in patients with OSMF for early detection of oral cancer. The literature has shown potential chance of takin saliva as a mode of diagnosis. For that individual markers would not be able to play any significant role. Therefore, a panel of markers from all types of constitutes need to be studied together to find a reliable method. For instance, taking electrolytes, inflammatory markers (C-reactive protein, IL6, IL8), Immunoglobulins (IgG, IgA), mRNAs, proteins and tumor markers and cells together would be a practical approach.

\section{Conclusion}

Saliva has a potential for use as a non-invasive mode of liquid biopsy and can be used as surveillance, screening and early detection of oral cancers in pre-malignant conditions where oral biopsy would further raise the risk of malignant transformation. However individual markers or the same group of markers might not be helpful thus taking a panel of all types of constituents would be appropriate approach.

\section{Acknowledgement}

None.

\section{Conflict of Interest}

No conflict of interest.

\section{References}

1. Arglebe C(1981) Biochemistry of human saliva. Adv Otorhinolaryngology 26: $97-234$

2. Pfaffe T, Cooper-White J, Peter B, Karam K, Chamindie P (2011) Diagnostic potential of saliva: current state and future applications. Clin Chem 57(5): 675-687.

3. Farnaud SJ, Ourania K, Stephen JG, Derek R (2010) Saliva: physiology and diagnostic potential in health and disease. ScientificWorldJournal 10: 434-456.

4. Nagler R, Dayan D (2006) The dual role of saliva in oral carcinogenesis. Oncology 71(1-2): 10-17.

5. Mohiuddin S, Fatima N, Hosein S (2016) High risk of malignant transformation of oral submucous fibrosis in Pakistani females: A potential national disaster. J Pak Med Assoc. 66(11): 1362-1366.

6. Assad DX, Elisa Cançado PM, Caroline LL, Porto de Toledo I, Hélène Chardin, et al. (2020) Salivary metabolites to detect patients with cancer: a systematic review. Int J Clin Oncol 25(6): 1016-1036.

7. Shpitzer T, Gideon Bahar, Raphael Feinmesser, Rafael M Nagler (2007) A comprehensive salivary analysis for oral cancer diagnosis. J Cancer Res Clin Oncol 133(9): 613-617.

8. Nagler R, Weizman A, Gavish A (2019) Cigarette smoke, saliva, the translocator protein $18 \mathrm{kDa}$ (TSPO), and oral cancer. Oral Dis 25(8): 1843-1849.

9. Nagler RM (2007) Altered salivary profile in heavy smokers and its possible connection to oral cancer. Int J Biol Markers 22(4): 274-280.

10. Giebułtowicz J, Wroczyński, Samolczyk-Wanyura D (2011) Comparison of antioxidant enzymes activity and the concentration of uric acid in the saliva of patients with oral cavity cancer, odontogenic cysts and healthy subjects. J Oral Pathol Med 40(9): 726-730.

11. Kallalli BN, Kamala Rawson, Muzammil, Ankur Singh, Mohammed Arif Awati, et al. (2016) Lactate dehydrogenase as a biomarker in oral cancer and oral submucous fibrosis. J Oral Pathol Med 45(9): 687-690. 
12. Ghallab NA, Shaker OG (2017) Serum and salivary levels of chemerin and MMP-9 in oral squamous cell carcinoma and oral premalignant lesions. Clin Oral Investig 21(3): 937-947.

13. Khyani IAM, Masood A Qureshi, Talat Mirza, M Umar Farooq (2017) Detection of interleukins- 6 and 8 in saliva as potential biomarkers of oral pre-malignant lesion and oral carcinoma: A breakthrough in salivary diagnostics in Pakistan. Pak J Pharm Sci 30(3): 817-823.

14. Achalli, S., Medhini Madi, Subhas G Babu, Shishir Ram Shetty, Suchetha Kumari, et al. (2017) Sialic acid as a biomarker of oral potentially malignant disorders and oral cancer. Indian J Dent Res 28(4): 395-399.

15. Awasthi N (2017) Role of salivary biomarkers in early detection of oral squamous cell carcinoma. Indian J Pathol Microbiol 60(4): 464-468.

16. Ishikawa S, David T W Wong, Masahiro Sugimoto, Frederico Omar Gleber-Netto, Feng Li, et al. (2019) Identification of salivary metabolites for oral squamous cell carcinoma and oral epithelial dysplasia screening from persistent suspicious oral mucosal lesions. Clin Oral Investig 23(9): 3557-3563.

17. Metzger K, G Angres, H Maier, WD (1995) Lehmann Lipoxygenase products in human saliva: patients with oral cancer compared to controls. Free Radic Biol Med 18(2): 185-194.

18. Li Y, Maie A R St John, Xiaofeng Zhou, Yong Kim, Uttam Sinha, et al. (2004) Salivary transcriptome diagnostics for oral cancer detection. Clin Cancer Res 10(24): 8442-8450.

19. Zimmermann BG, NJ Park, DT Wong (2007) Genomic targets in saliva Ann N Y Acad Sci 1098: 184-191.

20. Park NJ, Hui Zhou, David Elashoff, Bradley S Henson, Dragana A Kastratovic, et al. (2009) Salivary microRNA: discovery, characterization, and clinical utility for oral cancer detection. Clin Cancer Res 15(17): 5473-5477.

21. Momen-Heravi, F., AJ Trachtenberg, WP Kuo, YS Cheng (2014) Genomewide Study of Salivary MicroRNAs for Detection of Oral Cancer. J Dent Res 93(7 Suppl): 86s-93s.
22.Zahran F, D Ghalwash, O Shaker, K Al-Johani, C Scully (2015) Salivary microRNAs in oral cancer. Oral Dis 21(6): 739-747.

23. Shpitzer T, Y Hamzany, G Bahar, R Feinmesser, D Savulescu, et al. (2009) Salivary analysis of oral cancer biomarkers. Br J Cancer 101(7): 11941198.

24. Jou YJ, Chia-Der Lin, Chih-Ho Lai, Chao-Hsien Chen, Jung-Yie Kao, et al. (2010) Proteomic identification of salivary transferrin as a biomarker for early detection of oral cancer. Anal Chim Acta 681(1-2): 41-48.

25. Brinkmann O, Dragana A Kastratovic, Milovan V Dimitrijevic, Vitomir S Konstantinovic, Drago B Jelovac, et al. (2011) Oral squamous cell carcinoma detection by salivary biomarkers in a Serbian population. Oral Oncol 47(1): 51-55.

26. Elashoff D, Hui Zhou, Jean Reiss, Jianghua Wang, Hua Xiao, et al. (2012) Prevalidation of salivary biomarkers for oral cancer detection. Cancer Epidemiol Biomarkers Prev 21(4): 664-672.

27. Zubairy YF, Vinayak W Patil, Tabita Benjamin, Daya Jangam, Mohammed Nadeem Ahmed Bijle, et al., (2012) Effect of methylxanthines (coffee/ tea consumers) on oral precancer and oral cancer patients with smoking and smokeless tobacco habits. J Contemp Dent Pract 13(6): 745-758.

28. Panneer Selvam N, Sadaksharam J (2015) Salivary interleukin-6 in the detection of oral cancer and precancer. Asia Pac J Clin Onco 11(3): 236241.

29. Wei J, Guoxiang Xie, Zengtong Zhou, Peng Shi, Yunping Qiu, et al. (2011) Salivary metabolite signatures of oral cancer and leukoplakia. Int J Cancer 129(9): 2207-2217.

30. Geng XF, Meng Du, Jing-xiu Han, Min Zhang, Xiao-fei Tang, et al. (2013) Saliva CA125 and TPS levels in patients with oral squamous cell carcinoma. Int J Biol Marker 28(2): 216-220.

31. López-Verdín S, JJ Soto-Avila, AL Zamora-Perez, BP Lazalde-Ramos, ML Martínez-Fierro, et al. (2017) Patients with advanced oral squamous cell carcinoma have high levels of soluble E-cadherin in the saliva. Med Oral Patol Oral Cir Bucal 22(6): e694-e701. 